\title{
MRI-detected osteitis is not associated with the presence or level of ACPA alone, but with the combined presence of ACPA and RF
}

Debbie M. Boeters ${ }^{1 *}$, Wouter P. Nieuwenhuis ${ }^{1}$, Marije K. Verheul ${ }^{1}$, Elize C. Newsum ${ }^{1}$, Monique Reijnierse ${ }^{2}$, René E. M. Toes ${ }^{1}$, Leendert A. Trouw ${ }^{1}$ and Annette H. M. van der Helm-van Mil ${ }^{1}$

\begin{abstract}
Background: In rheumatoid arthritis (RA) bone marrow edema (BME, osteitis) and anti-citrullinated protein antibodies (ACPA) are associated with radiographic progression. ACPA have been associated with BME, but it is unknown if this association is confined to ACPA and BME. We performed cross-sectional analysis of the association of ACPA, rheumatoid factor (RF) and anti-carbamylated protein (anti-CarP) antibodies with BME and other types of inflammation (synovitis, tenosynovitis) detected by magnetic resonance imaging (MRI).

Methods: Disease-modifying antirheumatic drug (DMARD)-naïve patients with early arthritis $(n=589)$, included in the Leiden Early Arthritis Clinic cohort, underwent contrast-enhanced 1.5 T MRI of unilateral wrist, metacarpophalangeal and metatarsophalangeal-joints at baseline. BME, synovitis and tenosynovitis were scored by two readers. ACPA, rheumatoid factor (RF) and anti-CarP were determined at baseline.

Results: In univariable analyses ACPA-positive patients had higher BME scores than ACPA-negative patients (median 4.5 vs. 2.0, $p<0.001$ ), but not more synovitis and tenosynovitis. Also RF (median 3.75 vs. 2.0, $p<0.001$ ) and anti-CarP antibodies (median 3.5 vs. 2.5, $p=0.012$ ) were associated with higher BME scores. Because the autoantibodies were concomitantly present, analyses were stratified for the presence of different autoantibody combinations. ACPA-positive (ACPA+), RF-negative (RF-), anti-CarP-negative (anti-CarP-) patients did not have higher BME-scores than ACPA-negative (ACPA-), RF-, anti-CarP- patients. However ACPA+, RF-positive (RF+), antiCarP- patients and ACPA+, RF+, anti-CarP-positive (anti-CarP+) patients had higher BME scores than ACPA-, RF-, anti-CarP- patients (median 5.0 and 4.5 vs. 2.0, $p<0.001$ and $p<0.001$ ). ACPA levels were not associated with BME scores. Analyses within RA- and UA-patients revealed similar results.
\end{abstract}

Conclusions: The presence of ACPA alone or ACPA level was not statistically significantly associated with BME scores, but the combined presence of ACPA and RF was associated with more BME. This suggests an additive role of RF to ACPA in mediating osteitis.

Keywords: Rheumatoid arthritis, ACPA, RF, MRI, Bone marrow edema

\footnotetext{
*Correspondence: D.M.Boeters@lumc.nl

'Department of Rheumatology C1-R, Leiden University Medical Center, PO

Box 9600, Leiden 2300, RC, The Netherlands

Full list of author information is available at the end of the article
} 


\section{Background}

Rheumatoid arthritis (RA) is characterized by chronic inflammation of the joints that may result in progressive structural damage. Magnetic resonance imaging (MRI) detects inflammation sensitively [1]. Whereas synovitis and tenosynovitis can also be evaluated by other imaging modalities, such as ultrasound, MRI is the only modality that depicts bone marrow edema (BME). Histopathology studies in RA have shown that BME lesions consist of infiltration by leucocytes and an increased number of osteoclasts [2-4]. Therefore, BME has also been named osteitis. These data suggest a link between BME and structural damage in RA. Indeed, the importance of BME is supported by several studies showing that BME is a predictor of radiographic evidence of progression [5-13]. A recent study even showed that the persisting presence of BME is associated with an odds ratio (OR) of 60 for erosive progression at the same location [14].

In addition to BME, anti-citrullinated protein antibodies (ACPA) are also a strong predictor of radiographic progression [15-23]. Up to two-thirds of patients with RA harbor ACPA, as has been known for many years [24]. However, the underlying mechanism linking ACPA with a more severe disease progression with increased joint destruction is incompletely elucidated. Recent data suggest that ACPA influences bone resorption by directly activating osteoclasts [25]. The combination of these findings lead us to hypothesize that ACPA are associated with BME.

Other studies, including a small study that we performed previously suggest there is association between BME and ACPA [26, 27]. However, ACPA are often simultaneously present with other RA-related autoantibodies such as rheumatoid factor (RF) and anti-carbamylated protein antibodies (anti-CarP) (which also have been associated with radiographic destruction) [28-30]. To our knowledge the effects of different autoantibodies (either alone or in combination) on BME are unknown. Furthermore, the association between different autoantibodies and other types of inflammation detected by MRI (synovitis and tenosynovitis) has never been thoroughly explored. Therefore, this cross-sectional study aimed to investigate the associations of ACPA, RF and anti-CarP antibodies with BME, synovitis and tenosynovitis.

\section{Methods}

\section{Patients}

The 589 patients with early arthritis were consecutively included in the Leiden Early Arthritis Clinic (EAC) between 2010 and 2014. The EAC is an inception cohort that includes patients attending the rheumatologist who present with clinically confirmed arthritis and symptom duration of $<2$ years. Patients were disease-modifyingantirheumatic-drug (DMARD)-naïve at inclusion. The cohort started in 1993. MRI was performed from 2010 onwards; 598 patients underwent MRI, and 9 were excluded from analysis because no contrast agent was administered. The median interval between inclusion in the study and MRI was 1.3 weeks. Questionnaires were administered, and joint counts and blood samples were collected at baseline [31]. Baseline serum samples were tested for ACPA (anti-CCP2, Eurodiagnostica, Arnhem, the Netherlands, cutoff value $\geq 7 \mathrm{U} / \mathrm{ml}$ ), IgM RF (as described previously, in-house ELISA [32]) and IgG anti-CarP antibodies against carbamylated fetal calf serum (FCS). Anti-CarP was determined as described previously [28]; the cutoff for positivity for anti-CarP was based on the mean plus two times the standard deviation from a set of 204 healthy controls. One year after presentation, 183 patients fulfilled the 1987 criteria for RA [33], 214 had undifferentiated arthritis (UA) and 192 had other forms of arthritis, including psoriatic arthritis, reactive arthritis and others (Table 1).

\section{Magnetic resonance imaging and scoring}

At baseline, MRI was performed of the metacarpophalangeal (MCP), wrist and metatarsophalangeal (MTP) joints on the most painful side or on the dominant side in the case of symmetric symptoms. MRI was performed using an MSK Extreme 1.5 T extremity MRI system. In the wrist and MCP joints a coronal T1-weighted sequence was acquired before intravenous injection of contrast agent (gadoteric acid). Post-contrast, coronal and axial T1-weighted sequences with frequency-selective fat saturation were obtained. The forefoot was scanned using a T1-weighted sequence and a T2-weighted fat-saturated sequence in the axial plane. The protocol was shortened after 371 patients had been imaged [34]. For post-contrast imaging of the foot in the remaining 218 patients, T1-weighted, fat-saturated sequences were obtained in the coronal and axial plane and the T2-weighted sequence was deleted. A more detailed description of the scan protocol is provided elsewhere $[14,35,36]$ and in Additional file 1.

BME and synovitis were scored semi-quantitatively according to the rheumatoid arthritis MRI scoring system (RAMRIS) [37], with the exception that BME was assessed on a contrast-enhanced T1-weigthed fat-suppressed sequence. Previous studies have shown that T2-weighted fat-saturated sequences and contrast-enhanced T1-weighted fat-saturated images perform equally well in the depiction of BME [34, 38, 39], and according to the European Society of Musculoskeletal Radiology (ESSR), both sequences can be used to evaluate BME [40]. The T1-sequence was used as it allowed a shorter scan time. In addition, tenosynovitis in the wrist and MCP joints was scored according to the method proposed by Haavardsholm et al. [41], with tenosynovitis assessed for the flexor and extensor tendons of MCP joints $2-5$ using the same scale of $0-3$ as for the wrist. MR images 
Table 1 Baseline characteristics of the total group of patients with early arthritis and the subgroups of patients with rheumatoid arthritis (RA) or undifferentiated arthritis (UA)

\begin{tabular}{lll}
\hline Variable & $\begin{array}{l}\text { All patients with early arthritis } \\
(\mathrm{n}=589)\end{array}$ & $\begin{array}{l}\text { Subgroup of patients with RA or UA } \\
(\mathrm{n}=397)\end{array}$ \\
\hline Age, mean (sd) & $54.8(16)$ & $54.9(15)$ \\
Female, $n$ (\%) & $363(62)$ & $253(64)$ \\
Symptom duration, median (IQR), weeks & $12.6(5-27)$ & $12.2(5-26.2)$ \\
TJC, median (IQR) & $4(2-7)$ & $4(2-6)$ \\
SJC, median (IQR) & $3(2-7)$ & $3(2-7)$ \\
CRP (mg/L), median (IQR) & $5.7(3-17)$ & $6(3-17)$ \\
ACPA positivity, $n$ (\%) & $141(24)$ & $123(31)$ \\
RF positivity, $n$ (\%) & $193(33)$ & $151(38)$ \\
anti-CarP positivity, $n$ (\%) & $88(15)$ & $71(18)$ \\
Total RAMRIS, median (IQR) & $12.5(5.5-24)$ & $13.5(6-24)$ \\
Total BME score, median (IQR) & $2.5(1-6)$ & $2.5(1-6)$ \\
Total synovitis score, median (IQR) & $3.5(1-7.5)$ & $4(1.5-8)$ \\
Total tenosynovitis score, median (IQR) & $2(0-6)$ & $3(0.5-6)$
\end{tabular}

The diagnoses of the 589 patients with early arthritis were: 183 RA (according to the 1987 RA criteria), 214 UA, 14 reactive arthritis, 14 gout, 2 pseudogout, 30 psoriatic arthritis, 35 inflammatory osteoarthritis, 4 Lyme's arthritis, 1 paramalignant arthritis, 3 systemic lupus erythematosus, 11 other systemic disorder, 7 mixed connective tissue disease, vasculitis, 4 sarcoidosis, 9 spondyloarthritis, 8 remitting seronegative symmetrical synovitis with pitting edema, and 50 other unspecified conditions. $n$ number of patients, sd standard deviation, IQR interquartile range, symptom duration time between symptom onset and inclusion in cohort, $T J C$ 6 tender joint count, SJC 66 swollen joint count, CRP C-reactive protein, ACPA anti-citrullinated protein antibodies, $R F$ rheumatoid factor, anti-CarP anti-carbamylated protein antibodies, RA/UA subgroup of patients with rheumatoid arthritis (according to the 1987 RA criteria) or undifferentiated arthritis

were scored by two readers blinded to any clinical data. The mean total scores of both readers for BME, synovitis and tenosynovitis were used in further analyses. The intra-reader class correlation coefficients for the total inflammation score based on $40 \mathrm{MR}$ images that were scored twice, were 0.98 and 0.93, respectively. Based on all 598 scans, the interreader class correlation coefficient for the total inflammation score was 0.95 .

\section{Sensitivity analyses}

Our primary analyses were performed in all 589 early arthritis patients, as we hypothesized that direct association between autoantibodies and MRI-detected inflammation, if present, would be independent of the clinical diagnosis. However, analyses were repeated in the subgroup of 397 patients classified with UA or RA according to the 1987 American College of Rheumatology (ACR) criteria. Patients who had UA after one year were included in these analyses because misclassification could have occurred due to DMARD treatment during the first year that could have hampered progression to fulfilling the 1987 criteria for RA. As some of these patients with UA would have progressed to RA without treatment (but now remain unclassified), we also studied the patients with UA.

\section{Statistical analysis}

The $t$ test, multivariable linear regression, and multivariable logistic regression were used for analysis as appropriate. In multivariable linear regression analysis, the
BME scores were $\log _{10}$-transformed $\left(\log _{10}(\right.$ score +1$\left.)\right)$ to approximate a normal distribution. For interpretation, the obtained effect size (beta) was back-transformed to the normal score. All models were adjusted for age, gender and symptom duration. Baseline data on ACPA, $\mathrm{RF}$, and anti-CarP were dichotomized (seropositive vs. seronegative). Anti-CarP data were missing for 16 patients. ACPA and RF status was known for all patients. To determine the effect of ACPA levels on BME, baseline ACPA was categorized into three groups within ACPApositive patients based on the range of ACPA values (low, intermediate, or high); the thresholds were: $\geq 7 \mathrm{U} / \mathrm{ml}$, $\geq 167 \mathrm{U} / \mathrm{ml}$ and $\geq 327 \mathrm{U} / \mathrm{ml}$. $P$ values $\leq 0.05$ were considered significant. Analyses were performed using SPSS version 23.0 (IBM).

\section{Results}

\section{Baseline characteristics}

Baseline characteristics of the 589 patients are presented in Table 1.

\section{ACPA is associated with BME at baseline}

We first evaluated whether patients with ACPA $(n=141)$ or without ACPA $(n=448)$ had differences in BME scores (Fig. 1a). ACPA-positive patients had higher BME scores $($ median $=4.5)$ than ACPA-negative patients $($ median $=2.0$, $p<0.001$ ). We subsequently questioned whether ACPA is also associated with other types of MRI-detected inflammation, i.e., synovitis and tenosynovitis. There were no 


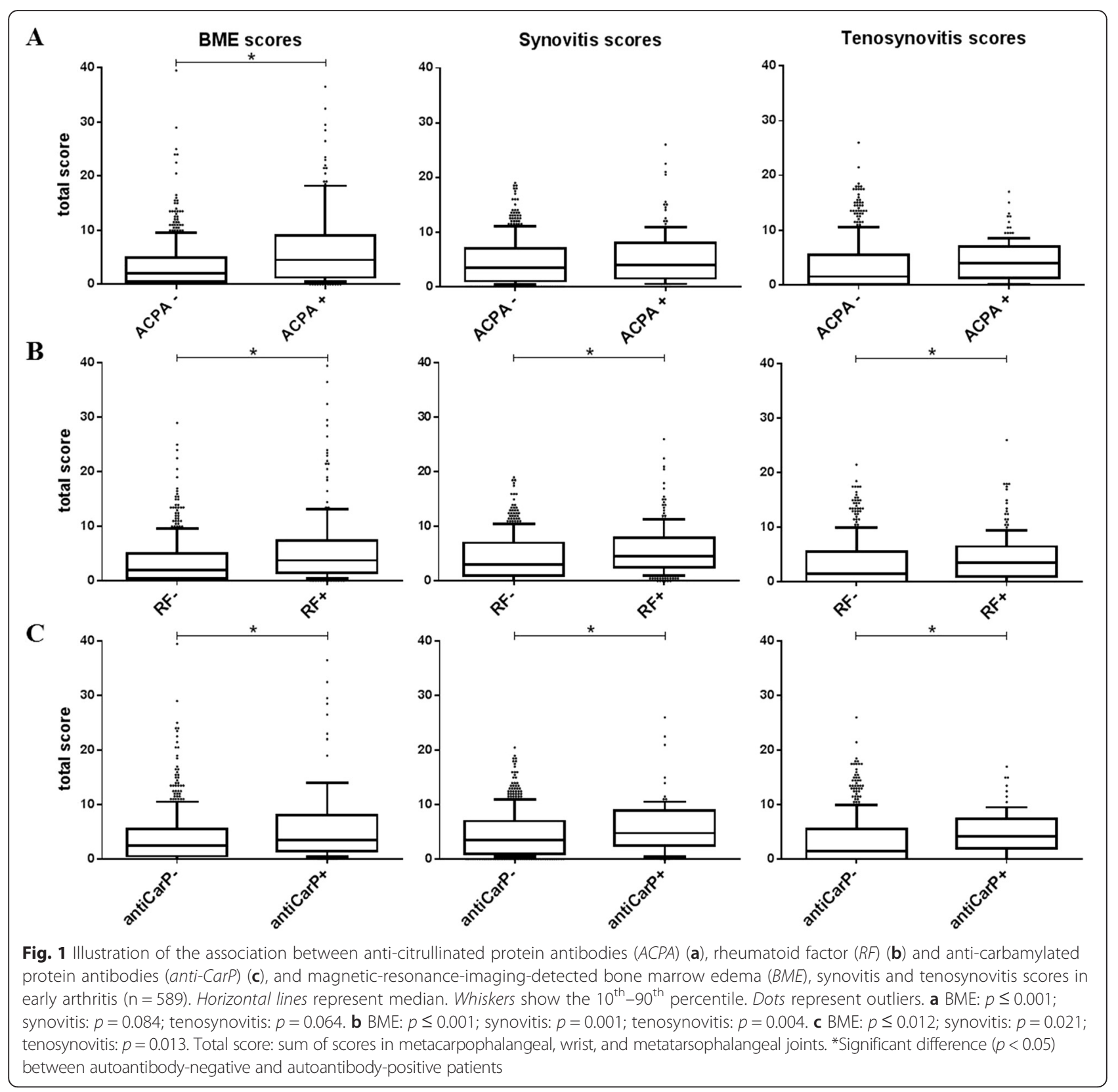

statistically significant differences in synovitis or tenosynovitis scores in ACPA-positive and ACPA-negative patients (Fig. 1a). Similar results were obtained for BME when only patients with RA and UA were studied (ACPA-positive median $=3.5$, ACPA-negative median $=2.0, p=0.001$ ) and no statistically significant differences were observed for synovitis and tenosynovitis (Additional file 2: Figure S1A). Based on these data ACPA seemed to be primarily associated with BME.

RF and anti-CarP antibodies are also associated with BME We were also interested in whether RF and anti-CarP antibodies are also associated with more severe BME.
The BME scores were higher in RF-positive patients (median $=3.75)$ compared to RF-negative patients (median $=2.0, p<0.001$, Fig. 1b). Similarly, BME scores were also higher in anti-CarP-positive than in anti-CarPnegative patients (median $=3.5$ vs. $2.5, p=0.012$, Fig. $1 \mathrm{c}$ ).

Besides BME, synovitis and tenosynovitis scores were also higher in RF-positive than in RF-negative patients (synovitis: median 4.5 vs. $3.0, \mathrm{p}=0.001$; tenosynovitis: median 3.5 vs. $1.5, p=0.004$ ). Synovitis and tenosynovitis scores were also higher in anti-CarP-positive than in anti-CarP-negative patients (synovitis: median $=4.75$ vs. $3.5, p=0.021$; tenosynovitis: median $=4.25$ vs. $1.5, p=$ 0.013). In patients with RA and UA only the BME scores 
were significantly higher in RF-positive $(\mathrm{RF}+)$ or antiCarP-positive (anti-CarP+) patients, but synovitis and tenosynovitis scores were not statistically significantly different (BME: RF+ median $=3.5$, RF-negative (RF-) median $=2.0, p=0.002$; anti-CarP + median $=3.5$, anti-CarPnegative (anti-CarP-) median $=2.5, p=0.017$, Additional file 2: Figure S1B, C).

Patients can concurrently have BME, synovitis, and tenosynovitis. To unravel the independent association between RF and BME, synovitis, and tenosynovitis scores, multivariable logistic regression analysis was performed with RF as the dependent variable and BME, synovitis, and tenosynovitis as independent variables. The same was done with anti-CarP as the dependent variable. In early arthritis, only the BME score was independently associated with RF $(p<0.001)$ or with anti-CarP $(p=0.003)$. Similar results were observed in the subgroup of patients with RA or UA, in whom only BME was associated with RF $(p<0.001)$ or with anti-CarP $(p=0.001)$. Thus, these multivariable analyses suggest that the BME score is independently associated with RF or anti-CarP, in contrast to the synovitis and tenosynovitis scores. Because of this result, and because it was observed that there was an association between BME and ACPA, subsequent analyses focused on BME.

ACPA and RF are both independently associated with BME Patients frequently have a combination of different types of inflammation, and also concomitantly have the three autoantibodies. For more insight into the relationship between the different autoantibodies and BME, multivariable linear regression analysis was performed with BME as the outcome and the three autoantibodies as independent variables. Both ACPA and RF were significantly associated with BME (ACPA: $p=0.015$, beta $=1.33$, indicating that ACPA-positive $(\mathrm{ACPA}+)$ patients had $33 \%$ higher $\mathrm{BME}$ scores than ACPA-negative (ACPA-) patients; RF: $p=0.004$, beta $=1.31$, indicating that $R F+$ patients had $31 \%$ higher BME scores than RF- patients). Additional adjustments for CRP and SJC produced similar results (ACPA: $p=0.009$, beta $=1.36$; RF: $p=0.001$, beta $=1.36$ ). In a similar analysis in the subgroup of patients with RA and UA, there was a trend towards significance for ACPA $(p=0.091$, beta $=$ 1.26) and a significant result for RF (RF: $p=0.022$, beta $=$ 1.31). Thus, together these data indicated that ACPA and $\mathrm{RF}$ are independently associated with BME scores.

Combined presence of ACPA and RF is associated with BME The multivariable analyses described above did not evaluate different effects for combinations of antibodies. Different autoantibody combinations were compared for more insight into the effect of individual antibodies and a combination of antibodies on BME (Fig. 2). In the absence of both RF and anti-CarP, ACPA was not associated

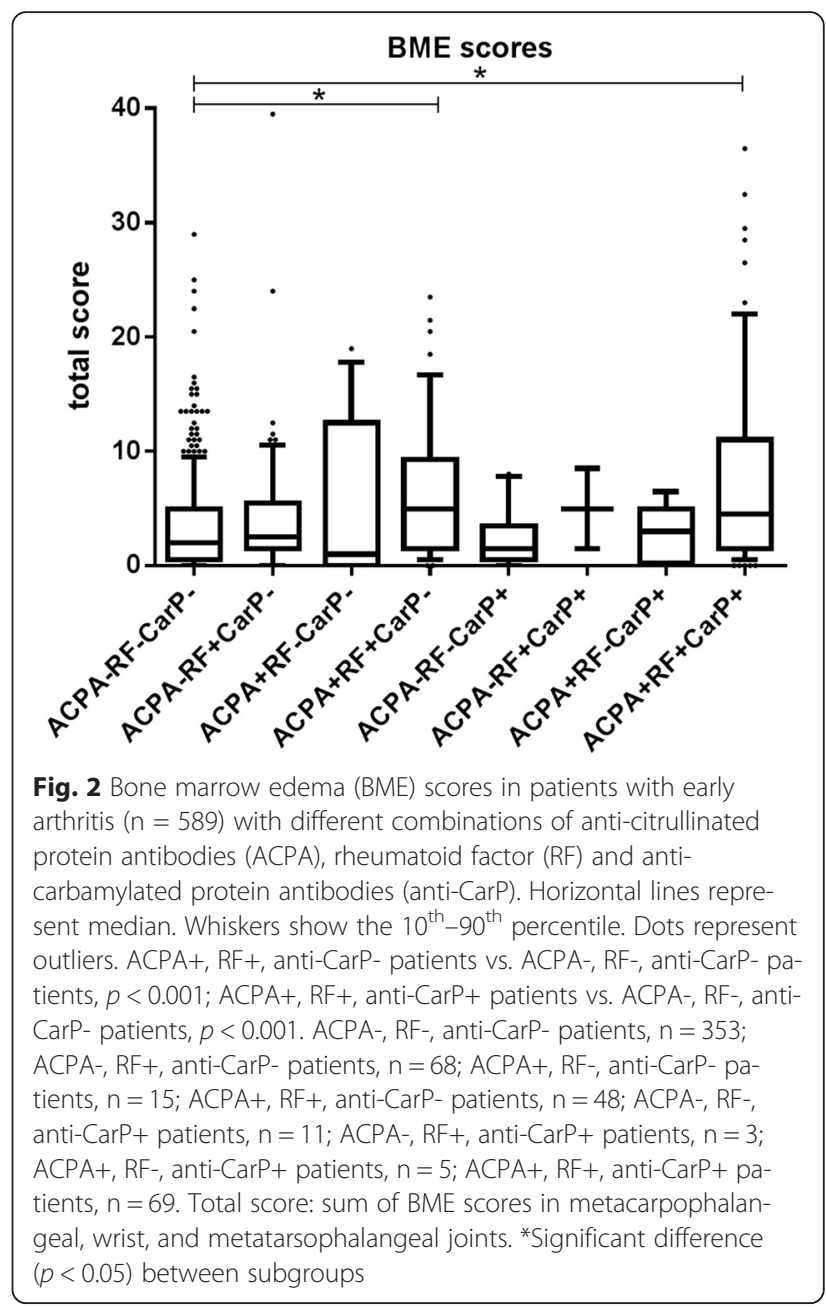

with BME (ACPA+, RF-, anti-CarP- patients vs. ACPA-, RF-, anti-CarP- patients, median 1.0 vs. $2.0, p=0.43$ ). Also the presence of RF or anti-CarP alone was not associated with BME (ACPA-, RF+, anti- CarP- patients and ACPA-, RF-, anti-CarP+ patients vs. ACPA-, RF-, anti-CarP- patients, median 2.5 and 1.5 vs 2.0 respectively, $p=0.096$ and $p=0.43)$. However $\mathrm{ACPA}+, \mathrm{RF}+$, anti-CarP-patients and $\mathrm{ACPA}+, \mathrm{RF}+$, anti-CarP+ patients did have significantly higher BME-scores than ACPA-, RF-, anti-CarPpatients (median 5.0 and 4.5 vs. 2.0 respectively, $p<0.001$ and $p<0.001)$. The same analysis in only RA- and UApatients showed that $\mathrm{ACPA}+, \mathrm{RF}+$, anti-CarP- patients and $\mathrm{ACPA}+, \mathrm{RF}+$, anti-CarP+ patients had higher $\mathrm{BME}-$ scores than ACPA-, RF-, anti-CarP- patients (median 4.5 and 4.5 vs. 2.0 respectively, $p<0.001$ and $p<0.001$, Fig. 3 ). Thus only the combined presence of ACPA and RF (with or without the presence of anti-CarP) was associated with higher BME-scores.

\section{ACPA level is not associated with BME}

In general, patients who carry different RA-related autoantibodies also have higher levels of ACPA [42]. In our 


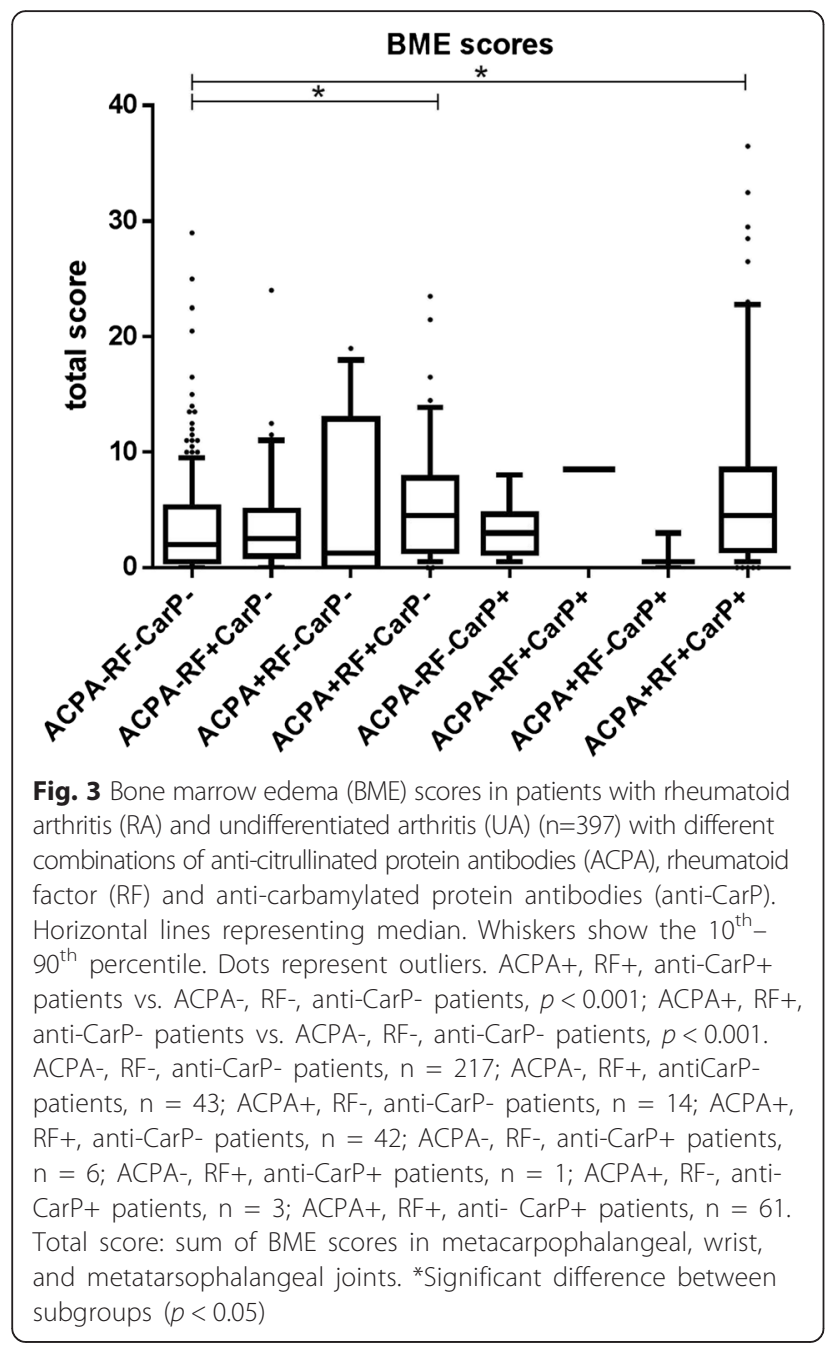

present data we also observed higher ACPA levels in patients who also carried RF and anti-CarP (ACPA+, RF-, antiCarP- patients median $116 \mathrm{U} / \mathrm{ml}, \mathrm{ACPA}+, \mathrm{RF}+$, anti-CarPpatients median $155 \mathrm{U} / \mathrm{ml}, \mathrm{ACPA}+$, RF-, anti-CarP+ patients median $92 \mathrm{U} / \mathrm{ml}, \mathrm{ACPA}+$, $\mathrm{RF}+$, anti-CarP+ patients median $257 \mathrm{U} / \mathrm{ml}, p=0.020$ ). This prompted us to explore whether the combined presence of ACPA and RF with higher BME scores could be explained by higher ACPA levels. To investigate the association between BME and ACPA levels, ACPA were studied as continuous data (Additional file 3: Figure S2) and divided into three subgroups. The BME scores observed in these ACPA categories were not different (Fig. 4). Similarly, no differences were observed when analyzing the BME scores in relation to ACPA levels in patients with RA and UA (Additional file 4: Figure S3). These data suggest that it is the combined presence of ACPA and RF that is associated with BME, rather than ACPA levels.
Combined presence of ACPA, RF and anti-CarP is associated with synovitis and tenosynovitis

The analyses focused on BME as the different autoantibodies were not associated with synovitis or tenosynovitis scores in univariable analyses (for ACPA) or in multivariable analyses (for RF and anti-CarP). However, having observed that higher BME scores were primarily associated with the combined presence of ACPA and RF (and not with the presence of a single antibody), we reasoned that it might also be possible that antibodies were not individually associated with synovitis or tenosynovitis scores, but that some combinations of autoantibodies were associated with synovitis or tenosynovitis scores. To study this, we finally assessed the association between different combinations of autoantibodies and synovitis and tenosynovitis (Fig. 5). ACPA+, RF+, anti-CarP+ patients had higher synovitis scores than ACPA-, RF-, anti-CarP- patients (median 5.0 vs. 3.0, $p=0.001$ ). For tenosynovitis, $\mathrm{ACPA}+, \mathrm{RF}+$, anti-CarP+ patients had higher scores than ACPA-, RF-, anti-CarP- patients (median 4.5 vs. 1.0, $p<0.001)$, and $\mathrm{ACPA}+, \mathrm{RF}+$, anti-CarP+ patients had higher scores than $\mathrm{ACPA}+, \mathrm{RF}+$, anti-CarP- patients (median 4.5 vs. $3.5 p=0.039$ ). Thus, the combined presence of ACPA, RF and anti-CarP was associated with the highest synovitis and tenosynovitis scores.

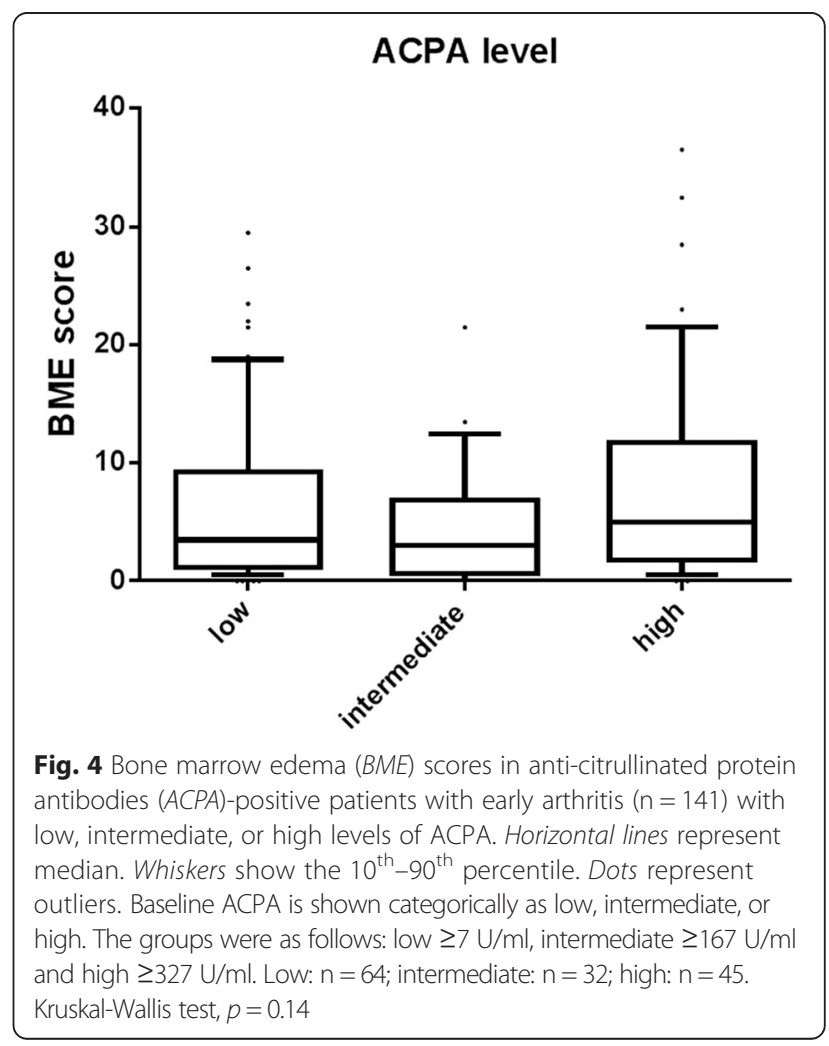



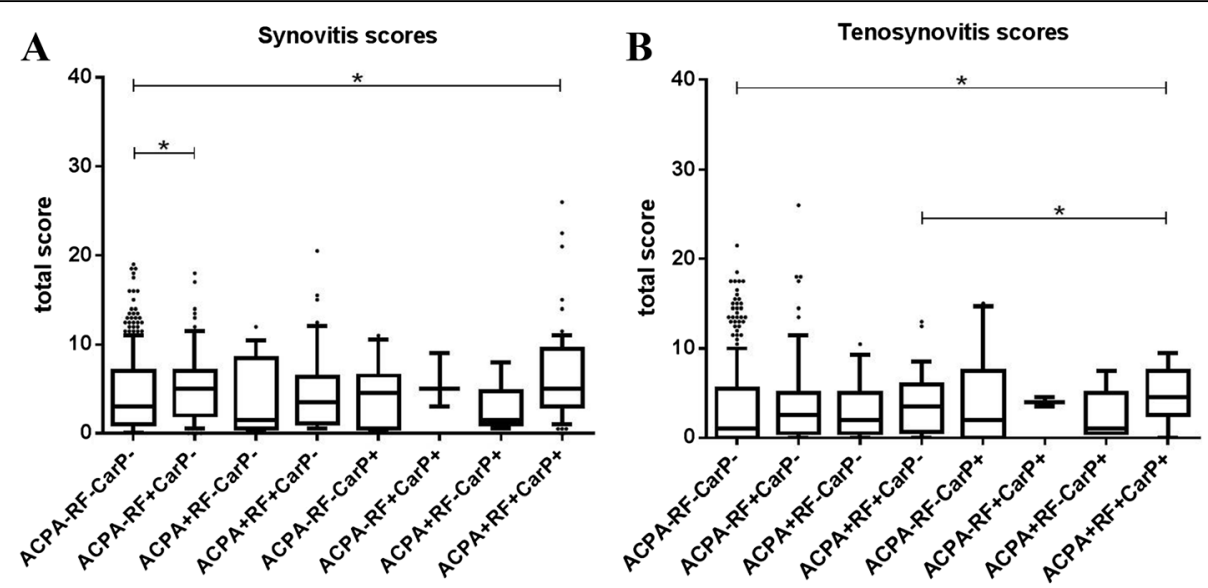

Fig. 5 Scores for synovitis detected by magnetic resonance imaging (a) and tenosynovitis (b) in patients with early arthritis ( $\mathrm{n}=589$ ) with different combinations of anti-citrullinated protein antibodies (ACPA), rheumatoid factor (RF) and anti-carbamylated protein antibodies (anti-CarP). Horizontal lines represent median. Whiskers show the $10^{\text {th }}-90^{\text {th }}$ percentile. Dots represent outliers. Synovitis: ACPA+, RF+, anti-CarP+ patients vs. ACPA-, RF-, anti-CarP- patients, $p<0.001$. Tenosynovitis: ACPA+, RF+, anti-CarP+ patients vs. ACPA-, RF-, anti-CarP- patients, $p<0.001$; ACPA+, RF+, anti-CarP+ patients vs. ACPA+, RF+, anti-CarP- patients, $p=0.039$. ACPA-, RF-, anti-CarP- patients, $n=353 ; A C P A-, R F+$, anti-CarP- patients, $n=69$; ACPA+, RF-, anti-CarP- patients, $n=15$; ACPA+, RF+, anti-CarP- patients, $n=48$; ACPA-, RF-, anti-CarP+ patients, $n=11$; ACPA-, RF+, anti-CarP+ patients, $\mathrm{n}=3$; ACPA+, RF-, anti-CarP+ patients, $\mathrm{n}=5$; ACPA+, RF+, anti-CarP+ patients, $\mathrm{n}=69$. Total score: sum of scores in metacarpophalangeal, wrist, and metatarsophalangeal joints. *Significant difference between subgroups $(p<0.05)$

\section{Discussion}

The relationship between ACPA and other RA-related autoantibodies and BME was subject of this study. We showed that ACPA, RF and anti-CarP antibodies were all associated with BME in univariable analyses. However, when the different autoantibody combinations were compared, the presence of ACPA alone was not associated with BME, but the combined presence of ACPA and RF (with or without anti-CarP) was associated with BME. The level of ACPA was not associated with BME, suggesting that this cannot be explained by ACPA levels but rather by the combined presence of ACPA and RF.

To our knowledge this is the first study including almost 600 MR images in which the relationship between the different autoantibodies and MRI-detected inflammation was investigated in detail. Due to this large sample size it was possible to evaluate the independent associations between BME and ACPA, RF and anti-CarP. Furthermore, it was possible to investigate the differential effects of the autoantibodies on the different types of MRI-detected inflammation. On analyses in subgroups of patients with different autoantibody combinations the BME scores were mainly increased when both ACPA and RF were present.

Our data suggest a potential interaction between RF and ACPA; however, the underlying mechanism by which ACPA and RF could act in concert was not studied. Potentially RF could have an immune-enhancing effect by crosslinking immune complexes and thereby activate monocytes or macrophages and induce cytokine expression. This is supported by a recent study that showed that RF augments TNFa production by ACPA immune complexes in vitro [43]. Another explanation could be that RF has a role in immune complex stabilization. ACPA bind to target antigens with low avidity but it could well be that when RF is also involved in the immune complex this binding is more stable. Further fundamental studies should be performed for more insight into the interaction between these two autoantibodies.

This study investigated local inflammation as observed on MRI. Recently the combined effect of ACPA and RF on systemic inflammation was investigated in RA, showing that the combined presence of ACPA and RF was associated with higher levels of pro-inflammatory cytokines and increased acute phase reactants and disease activity [43]. We also analyzed the association between the different autoantibody combinations and CRP, erythrocyte sedimentation rate (ESR), SJC and 28joint-count disease activity score (DAS28) as measures of disease activity in our patients with RA or UA at baseline; no large differences were observed but patients positive for all three autoantibodies had the highest disease activity scores (Additional file 5: Figure S4).

Association between the combined presence of autoantibodies and BME was observed. Since BME is associated with erosive progression [5-14], it would be interesting to investigate whether combinations of autoantibodies are also associated with radiographic progression. The association between ACPA or RF and radiographic progression is well-investigated [15-23]. However the number of studies investigating the combined effect of ACPA and RF is limited. A recent study 
in two cohorts showed no additive effect of RF on radiographic progression in ACPA-positive patients [44]. Another study analyzing high-resolution peripheral quantitative computed tomography (CT) images of the MCP joints in patients with RA showed that there was an additive effect of ACPA and RF on erosion number and size [45]. The read-out of these studies (microCT and conventional radiography) was different. It would be interesting to further unravel the association between different autoantibody combinations and erosive progression in further observational studies.

A limitation of the subgroup analysis is that some autoantibody combinations were infrequent and so no definite conclusions can be drawn for these. For instance, patients who were ACPA+, RF-, anti-CarP- were infrequent. Despite the limited power, there was no tendency in the data towards higher BME scores in these patients compared to the triple-negative group. This study is not the first that did not identify a deleterious effect of the presence of ACPA alone. Two recent papers reported on mice that were injected with ACPA, and although ACPA was detected in the joint, no signs of inflammation were observed in the synovium [46, 47]. Surprisingly, in our data the presence of ACPA alone even had a nonsignificant tendency towards a protective effect against synovitis (in all patients with early arthritis patients and in patients with RA or UA). Interestingly, two recent studies in humans showed that the presence of ACPA without RF was associated with lower disease activity $[42,43]$. In summary, further larger studies are needed to determine the role of ACPA single positivity.

Another limitation could be that we used contrastenhanced T1-weighted images to assess BME. Using the RAMRIS method, T2-weighted fat-suppressed sequences, or when this sequence is not available, a short tau inversion recovery (STIR) sequence, should be used to assess BME. However, it has been demonstrated that a contrastenhanced T1-weighted fat-suppressed sequence performed equally well to depict BME as a T2-weighted fatsuppressed sequence [34, 38, 39] and the evaluation of BME on a T1-weighted fat suppressed sequence is also supported by the ESSR [40]. In this study the contrastenhanced T1-weighted fat-suppressed sequence was used because it allowed a shorter scan time and has a higher signal-to-noise ratio [34, 38].

A third limitation is that the scan protocol for the foot was changed. When the analyses of the different autoantibody combinations and BME scores in patients with early arthritis were repeated separately in the patients scanned with or without the coronal sequence of the foot, the presence of ACPA alone was not associated with higher BME scores, but the combined presence of ACPA and RF was associated with higher BME scores (data not shown). This suggests that the change in scan protocol for the foot had no major influences on the results of this study.

Finally, our arthritis cohort includes patients with early disease who presented with different diagnoses. We hypothesized that direct association between ACPA and MRI-detected inflammation would be independent of the clinical diagnosis. However, to exclude an effect of this heterogeneity in patient selection on our findings we repeated all analyses within the subgroup of patients with RA and UA. This produced similar results.

Of note, the differences observed in BME scores were statistically significant but the absolute differences were relatively small. The variation in BME scores was only partly explained by the autoantibody status. Nonetheless the present study does increase our understanding of the relationship between autoantibodies and BME, which are both predictors of radiographic progression. The observation that ACPA is associated with osteitis, only when RF is present, fuels further laboratory studies on the biological relevance of these autoantibodies.

\section{Conclusions}

In conclusion, the presence of ACPA alone and ACPA serum levels were not associated with BME scores. However, BME scores were higher when patients were seropositive for both ACPA and RF. These results suggest that RF has an additive role to ACPA in mediating osteitis.

\section{Additional files}

Additional file 1: MRI protocol. (DOC $36 \mathrm{~kb}$ )

Additional file 2: Figure S1. Illustration of association between ACPA (A), RF (B) and anti-CarP (C) and MRI-detected BME, synovitis and tenosynovitis scores in patients with RA and UA $(n=397)$. Horizontal lines represent median. Whiskers show the $10^{\text {th }}-90^{\text {th }}$ percentile. Dots represent outliers. A BME: $p=0.001$; synovitis: $p=0.776$; tenosynovitis: $p=0.99$. B BME: $p=0.002$; synovitis: $p=0.19$; tenosynovitis: $p=0.26$. C BME: $p=0.017$; synovitis: $p=0.085$; tenosynovitis: $p=0.056$. Total score: sum of scores in MCP, wrist, and MTP joints. MRI magnetic resonance imaging, ACPA anti-citrullinated protein antibodies, RF rheumatoid factor, anti-CarP anti-carbamylated protein antibodies, $B M E$ bone marrow edema. *Significant difference between autoantibodynegative and autoantibody-positive patients ( $p<0.05)$. (JPG $76 \mathrm{~kb}$ )

Additional file 3: Figure S2. Association between ACPA level and BME scores. Association between ACPA level and BME scores within ACPA-positive patients with early arthritis $(\mathbf{A})(\mathrm{n}=141, r=0.071, p=0.403)$ and within ACPA-positive patients with RA or UA (B) $(n=123, r=0.034, p=0.706)$. (JPG $24 \mathrm{~kb}$ )

Additional file 4: Figure S3. BME scores of ACPA-positive patients with RA or UA $(n=123)$ with low, intermediate, or high levels of ACPA. Horizontal lines represent median. Whiskers show the $10^{\text {th }}-90^{\text {th }}$ percentile. Dots represent outliers. Baseline ACPA levels are shown categorically as low, intermediate, or high. The groups were as follows: low $\geq 7 \mathrm{U} / \mathrm{ml}$, intermediate $\geq 167 \mathrm{U} / \mathrm{ml}$, and high $\geq 327 \mathrm{U} / \mathrm{ml}$. Low: $\mathrm{n}=57$; intermediate: $\mathrm{n}=27$; high: $\mathrm{n}=39$. ACPA anti-citrullinated protein antibodies, BME bone marrow edema. Kruskal-Wallis test, $p=0.23$. (JPG $19 \mathrm{~kb})$

Additional file 5: Figure S4. Measures of disease activity in patients with RA and UA ( $n=397$ ) with different combinations of ACPA, RF and anti-CarP. Association between CRP (A), ESR (B), SJC (C) and DAS28 (D) 
and different autoantibody combinations. Horizontal lines represent median. Whiskers show the $10^{\text {th }}-90^{\text {th }}$ percentile. Dots represent outliers. ACPA anti-citrullinated protein antibodies; RF: rheumatoid factor; anti-CarP: anti-carbamylated protein antibodies, CRP C-reactive protein, ESR erythrocyte sedimentation rate, SJC swollen joint count based on 66 joints, DAS28 disease activity score in 28 joints. ${ }^{*}$ Significant difference between subgroups $(p<0.05)$. (JPG $104 \mathrm{~kb})$

\section{Abbreviations}

ACPA, anti-citrullinated protein antibodies; anti-CarP, anti-carbamylated protein antibodies; BME, bone marrow edema; CRP, C-reactive protein; DMARD, disease-modifying-antirheumatic-drug; EAC, Early Arthritis Clinic; ELISA, enzyme-linked immunosorbent assay; ESR, erythrocyte sedimentation rate; ESSR, European Society of Musculoskeletal Radiology; FCS, fetal calf serum; $I Q R$, interquartile range; MCP, metacarpophalangeal; MRI, magnetic resonance imaging; MTP, metatarsophalangeal ; OR, odds ratio; RA, rheumatoid arthritis; RAMRIS, rheumatoid arthritis magnetic resonance imaging scoring system; RF, rheumatoid factor; SD, standard deviation; SJC, swollen joint count; TJC, tender joint count; TNF, tumor necrosis factor; UA, undifferentiated arthritis

\section{Acknowledgements}

Not applicable.

\section{Funding}

This work was supported by the Dutch Arthritis Foundation and the Netherlands Organization for Health Research and Development (Vidi grant). The funding sources had no role in the design and conduct of the study; collection, management, analysis, and interpretation of the data; preparation, review, or approval of the manuscript; or decision to submit the manuscript for publication.

\section{Availability of data and materials}

Data can be requested from the corresponding author.

\section{Authors' contributions}

DMB, WPN, and AHMvdHvM contributed to the conception and study design. DMB analyzed the data. DMB, WPN, MKV, MR, REMT, LAT, and AHMvdHvM contributed to interpretation of the data. ECN contributed to acquisition of the data. DMB and AHMvdHvM wrote the first version of the manuscript and DMB, WPN, MKV, ECN, MR, REMT, LAT, and AHMvdHvM revised it critically. DMB, WPN, MKV, ECN, MR, REMT, LAT, and AHMvdHvM read and approved the final manuscript.

\section{Authors' information}

Not applicable.

\section{Competing interests}

The authors declare that they have no competing interests.

\section{Consent for publication}

Not applicable.

\section{Ethics approval and consent to participate}

The study was approved by the medical ethics committee of the Leiden University Medical Center, which is named Commissie Medische Ethiek (CME). All patients signed informed consent.

\section{Author details}

'Department of Rheumatology C1-R, Leiden University Medical Center, PO Box 9600, Leiden 2300, RC, The Netherlands. ${ }^{2}$ Department of Radiology, Leiden University Medical Center, Leiden, The Netherlands.

Received: 13 May 2016 Accepted: 14 July 2016

Published online: 02 August 2016

\section{References}

1. McQueen F, Stewart N, Crabbe J, Robinson E, Yeoman S, Tan P, et al. Magnetic resonance imaging of the wrist in early rheumatoid arthritis reveals a high prevalence of erosions at four months after symptom onset. Ann Rheum Dis. 1998;57:350-6.
2. McQueen FM. Bone marrow edema and osteitis in rheumatoid arthritis: the imaging perspective. Arthritis Res Ther. 2012;14:224.

3. Jimenez-Boj E, Nöbauer-Huhmann I, Hanslik-Schnabel B, Dorotka R, Wanivenhaus A-H, Kainberger $\mathrm{F}$, et al. Bone erosions and bone marrow edema as defined by magnetic resonance imaging reflect true bone marrow inflammation in rheumatoid arthritis. Arthritis Rheum. 2007;56:1118-24.

4. Dalbeth N, Smith T, Gray S, Doyle A, Antill P, Lobo M, et al. Cellular characterisation of magnetic resonance imaging bone oedema in rheumatoid arthritis; implications for pathogenesis of erosive disease. Ann Rheum Dis. 2009;68:279-82.

5. Lisbona MP, Pàmies A, Ares J, Almirall M, Navallas M, Solano A, et al. Association of bone edema with the progression of bone erosions quantified by hand magnetic resonance imaging in patients with rheumatoid arthritis in remission. J Rheumatol. 2014;41:1623-9.

6. McQueen FM, Benton N, Perry D, Crabbe J, Robinson E, Yeoman S, et al. Bone edema scored on magnetic resonance imaging scans of the dominant carpus at presentation predicts radiographic joint damage of the hands and feet six years later in patients with rheumatoid arthritis. Arthritis Rheum. 2003:48:1814-27.

7. Gandjbakhch F, Foltz V, Mallet A, Bourgeois P, Fautrel B. Bone marrow oedema predicts structural progression in a 1-year follow-up of 85 patients with RA in remission or with low disease activity with lowfield MRI. Ann Rheum Dis. 2011;70:2159-62.

8. Palosaari K, Vuotila J, Takalo R, Jartti A, Niemelä RK, Karjalainen A, et al. Bone oedema predicts erosive progression on wrist MRI in early RA-a 2-yr observational MRI and NC scintigraphy study. Rheumatology. 2006;45:1542-8.

9. Haavardsholm EA, Bøyesen P, Østergaard M, Schildvold A, Kvien TK. Magnetic resonance imaging findings in 84 patients with early rheumatoid arthritis: bone marrow oedema predicts erosive progression. Ann Rheum Dis. 2008;67:794-800.

10. McQueen F, Stewart N, Crabbe J, Robinson E, Yeoman S, Tan P, et al. Magnetic resonance imaging of the wrist in early rheumatoid arthritis reveals progression of erosions despite clinical improvement. Ann Rheum Dis. 1999:58:156-63.

11. Hetland ML, Ejbjerg B, Hørslev-Petersen K, Jacobsen S, Vestergaard A, Jurik AG, et al. MRI bone oedema is the strongest predictor of subsequent radiographic progression in early rheumatoid arthritis. Results from a 2-year randomised controlled trial (CIMESTRA). Ann Rheum Dis. 2009:68:384-90.

12. Bøyesen $P$, Haavardsholm EA, Østergaard $M$, van der Heijde D, Sesseng $S$, Kvien TK. MRI in early rheumatoid arthritis: synovitis and bone marrow oedema are independent predictors of subsequent radiographic progression. Ann Rheum Dis. 2011;70:428-33.

13. Mundwiler ML, Maranian P, Brown DH, Silverman JM, Wallace D, Khanna D, et al. The utility of MRI in predicting radiographic erosions in the metatarsophalangeal joints of the rheumatoid foot: a prospective longitudinal cohort study. Arthritis Res Ther. 2009;11:R94.

14. Nieuwenhuis WP, van Steenbergen HW, Stomp W, Stijnen T, Huizinga TWJ, Bloem JL, et al. The Course of Bone Marrow Edema in Early Undifferentiated Arthritis and Rheumatoid Arthritis: A Longitudinal Magnetic Resonance Imaging Study at Bone Level. Arthritis Rheumatol. 2016;68:1080-8.

15. Kroot E-JJA, De Jong BAW, Van Leeuwen MA, Swinkels H, Van Den Hoogen FHJ, Van't Hof M, et al. The prognostic value of anti-cyclic citrullinated peptide antibody in patients with recent-onset rheumatoid arthritis. Arthritis Rheum. 2000;43:1831-5.

16. Meyer O, Labarre C, Dougados M, Goupille P, Cantagrel A, Dubois A, et al. Anticitrullinated protein/peptide antibody assays in early rheumatoid arthritis for predicting five year radiographic damage. Ann Rheum Dis. 2003;62:120-6.

17. Vencovsky J, Machacek S, Sedova L, Kafkova J, Gatterova J, Pesakova V, et al. Autoantibodies can be prognostic markers of an erosive disease in early rheumatoid arthritis. Ann Rheum Dis. 2003;62:427-30.

18. De Rycke L, Peene I, Hoffman I, Kruithof E, Union A, Meheus L, et al. Rheumatoid factor and anticitrullinated protein antibodies in rheumatoid arthritis: diagnostic value, associations with radiological progression rate, and extra-articular manifestations. Ann Rheum Dis. 2004;63:1587-93.

19. Forslind K, Ahlmen M, Eberhardt K, Hafstrom I, Svensson B. Prediction of radiological outcome in early rheumatoid arthritis in clinical practice: role of antibodies to citrullinated peptides (anti-CCP). Ann Rheum Dis. 2004; 63:1090-5.

20. van der Helm-van Mil AH, Verpoort KN, Breedveld FC, Toes RE, Huizinga TW. Antibodies to citrullinated proteins and differences in clinical progression of rheumatoid arthritis. Arthritis Res Ther. 2005;7:R949-58. 
21. Machold KP, Stamm TA, Nell VPK, Pflugbeil S, Aletaha D, Steiner G, et al. Very recent onset rheumatoid arthritis: clinical and serological patient characteristics associated with radiographic progression over the first years of disease. Rheumatology. 2007:46:342-9.

22. Lindqvist E, Eberhardt K, Bendtzen K, Heinegard D, Saxne T. Prognostic laboratory markers of joint damage in rheumatoid arthritis. Ann Rheum Dis. 2005;64:196-201.

23. Berglin E, Johansson T, Sundin U, Jidell E, Wadell G, Hallmans G. Radiological outcome in rheumatoid arthritis is predicted by presence of antibodies against cyclic citrullinated peptide before and at disease onset, and by IgA-RF at disease onset. Ann Rheum Dis. 2006;65:453-8.

24. Schellekens GA, de Jong BA, van den Hoogen FH, van de Putte LB, van Venrooij WJ. Citrulline is an essential constituent of antigenic determinants recognized by rheumatoid arthritis-specific autoantibodies. J Clin Invest. 1998;101:273-81.

25. Harre U, Georgess D, Bang H, Bozec A, Axmann R, Ossipova E, et al. Induction of osteoclastogenesis and bone loss by human autoantibodies against citrullinated vimentin. J Clin Invest. 2012;122:1791-802.

26. Tamai M, Kawakami A, Uetani M, Takao S, Tanaka F, Nakamura H, et al. The presence of anti-cyclic citrullinated peptide antibody is associated with magnetic resonance imaging detection of bone marrow oedema in early stage rheumatoid arthritis. Ann Rheum Dis. 2006;65:133-4.

27. Stomp W, Krabben A, van der Heijde D, Huizinga TWJ, Bloem JL, Mil AHM van der $\mathrm{H}$, et al. Are rheumatoid arthritis patients discernible from other early arthritis patients using 1.5 T extremity magnetic resonance imaging? A large cross-sectional study. J Rheumatol. 2014;41:1630-7.

28. Shi J, Knevel R, Suwannalai P, van der Linden MP, Janssen GMC, van Veelen PA, et al. Autoantibodies recognizing carbamylated proteins are present in sera of patients with rheumatoid arthritis and predict joint damage. Proc Natl Acad Sci USA. 2011;108:17372-7

29. Heide AVD, Remme CA, Hofman DM, Jacobs JWG, Bijlsma JWJ. Prediction of progression of radiologic damage in newly diagnosed rheumatoid arthritis. Arthritis Rheum. 1995;38:1466-74.

30. Aletaha D, Alasti F, Smolen JS. Rheumatoid factor determines structural progression of rheumatoid arthritis dependent and independent of disease activity. Ann Rheum Dis. 2013;72:875-80.

31. de Rooy DPC, van der Linden MPM, Knevel R, Huizinga TWJ, Mil AHM van der H. Predicting arthritis outcomes - what can be learned from the Leiden Early Arthritis Clinic? Rheumatology. 2011;50:93-100.

32. van der Linden MPM, Batstra MR, Bakker-Jonges LE, Foundation for Quality Medical Laboratory Diagnostics, Detert J, Bastian H, et al. Toward a datadriven evaluation of the 2010 American College of Rheumatology/European League Against Rheumatism criteria for rheumatoid arthritis: is it sensible to look at levels of rheumatoid factor? Arthritis Rheum. 2011;63:1190-9.

33. Arnett FC, Edworthy SM, Bloch DA, Mcshane DJ, Fries JF, Cooper NS, et al. The American Rheumatism Association 1987 revised criteria for the classification of rheumatoid arthritis. Arthritis Rheum. 1988:31:315-24.

34. Stomp W, Krabben A, van der Heijde D, Huizinga TWJ, Bloem JL, van der Helm-van Mil AHM, et al. Aiming for a shorter rheumatoid arthritis MRI protocol: can contrast-enhanced MRI replace T2 for the detection of bone marrow oedema? Eur Radiol. 2014;24:2614-22.

35. Steenbergen HW van, Mangnus L, Reijnierse M, Huizinga TWJ, Mil AHM van der H. Clinical factors, anticitrullinated peptide antibodies and MRI-detected subclinical inflammation in relation to progression from clinically suspect arthralgia to arthritis. Ann Rheum Dis. 2015. doi:10.1136/annrheumdis-2015208138.

36. Nieuwenhuis WP, Krabben A, Stomp W, Huizinga TWJ, van der Heijde D, Bloem $J$, et al. Evaluation of magnetic resonance imaging-detected tenosynovitis in the hand and wrist in early arthritis. Arthritis Rheumatol Hoboken NJ. 2015;67:869-76.

37. Østergaard M, Peterfy C, Conaghan P, McQueen F, Bird P, Ejbjerg B, et al OMERACT Rheumatoid Arthritis Magnetic Resonance Imaging Studies. Core set of MRI acquisitions, joint pathology definitions, and the OMERACT RA-MRI scoring system. J Rheumatol. 2003:30:1385-6.

38. Schmid MR, Hodler J, Vienne P, Binkert CA, Zanetti M. Bone marrow abnormalities of foot and ankle: STIR versus T1-weighted contrast-enhanced fat-suppressed spin-echo MR imaging. Radiology. 2002;224:463-9.

39. Mayerhoefer ME, Breitenseher MJ, Kramer J, Aigner N, Norden C, Hofmann S. STIR vs. T1-weighted fat-suppressed gadolinium-enhanced MRI of bone marrow edema of the knee: computer-assisted quantitative comparison and influence of injected contrast media volume and acquisition parameters. J Magn Reson Imaging. 2005;22:788-93.
40. Sudoł-Szopińska I, Jurik AG, Eshed I, Lennart J, Grainger A, Østergaard M, et al. Recommendations of the ESSR Arthritis Subcommittee for the Use of Magnetic Resonance Imaging in Musculoskeletal Rheumatic Diseases. Semin Musculoskelet Radiol. 2015:19:396-411.

41. Haavardsholm EA, Østergaard M, Ejbjerg BJ, Kvan NP, Kvien TK. Introduction of a novel magnetic resonance imaging tenosynovitis score for rheumatoid arthritis: reliability in a multireader longitudinal study. Ann Rheum Dis. 2007;66:1216-20.

42. Aletaha D, Alasti F, Smolen JS. Rheumatoid factor, not antibodies against citrullinated proteins, is associated with baseline disease activity in rheumatoid arthritis clinical trials. Arthritis Res Ther. 2015;17:229.

43. Sokolove J, Johnson DS, Lahey LJ, Wagner CA, Cheng D, Thiele GM, et al. Rheumatoid factor as a potentiator of anti-citrullinated protein antibody mediated inflammation in rheumatoid arthritis. Arthritis Rheumatol Hoboken NJ. 2014;66:813-21.

44. van Steenbergen HW, Ajeganova S, Forslind K, Svensson B, van der Helm-van Mil AHM. The effects of rheumatoid factor and anticitrullinated peptide antibodies on bone erosions in rheumatoid arthritis. Ann Rheum Dis. 2015;74:e3.

45. Hecht C, Englbrecht M, Rech J, Schmidt S, Araujo E, Engelke K, et al. Additive effect of anti-citrullinated protein antibodies and rheumatoid factor on bone erosions in patients with RA. Ann Rheum Dis. 2015;74:2151-6.

46. Wigerblad G, Bas DB, Fernades-Cerqueira C, Krishnamurthy A, Nandakumar KS, Rogoz K, et al. Autoantibodies to citrullinated proteins induce joint pain independent of inflammation via a chemokinedependent mechanism. Ann Rheum Dis. 2016;75:730-8.

47. Krishnamurthy A, Joshua V, Haj Hensvold A, Jin T, Sun M, Vivar N, et al. Identification of a novel chemokine-dependent molecular mechanism underlying rheumatoid arthritis-associated autoantibodymediated bone loss. Ann Rheum Dis. 2016;75:721-9.

\section{Submit your next manuscript to BioMed Central and we will help you at every step:}

- We accept pre-submission inquiries

- Our selector tool helps you to find the most relevant journal

- We provide round the clock customer support

- Convenient online submission

- Thorough peer review

- Inclusion in PubMed and all major indexing services

- Maximum visibility for your research

Submit your manuscript at www.biomedcentral.com/submit
C) Biomed Central 